\title{
Linkages between workplace stressors and quality of care from health professionals' perspective - Macedonian experience
}

\author{
Jovanka Karadzinska-Bislimovska*, Vera Basarovska, \\ Dragan Mijakoski, Jordan Minov, Sasho Stoleski, Nada Angeleska \\ and Aneta Atanasovska \\ Institute of Occupational Health of RM, WHO Collaborating Center, Skopje, \\ Republic of Macedonia
}

Objectives. During last two decades, within the process of transition, the socioeconomic reforms in Republic of Macedonia reflected on the national health care system. The objective of this article was to identify workplace stressors and factors that influence quality of care, from the perspective of health professionals (HPs), and to understand how they were linked in the context of such social circumstances.

Methods. A qualitative research based on focus group (FG) methodology was conducted in a general teaching hospital. Two main topics were the subjects of discussion in FGs: workplace stressors and factors that influence quality of care, from the HPs perspective. Six FGs were conducted with a total of 56 HPs (doctors, nurses, interns, and residents) divided into two sets of three FGs for each topic separately. Two sets of data were processed with thematic analysis, and the obtained results were compared with each other.

Results. By processing the data, we identified themes relating to factors that generate stress among HPs and factors that influence quality of care, from HPs' perspective. By comparing the two sets of themes, we found that many of them were identical, which means factors that increase workplace stress at the same time reduce quality of care.

Conclusions. Implementation of specific organizational interventions in the hospital setting can lead to the prevention of work-related stress and improvement in quality of care. Our research suggests that the prevention of work-related stress will impact positively on the quality of care, which may contribute to establish criteria and recommendations for the improvement in organizational culture and climate in hospitals.

*Correspondence should be addressed to Jovanka Karadzinska-Bislimovska, Institute of Occupational Health of RM, WHO Collaborating Center, II Makedonska Brigada 43, Skopje, Republic of Macedonia (e-mail: bislimovska_j@yahoo.com). 


\section{Statement of contribution}

\section{What is already known on this subject?}

- Psychosocial stress at work among health professionals is often present and well studied, but relations between job stress and quality of care were rarely examined.

- Job demands-resources model by Demerouti, Bakker, Nachreiner and Schaufeli (200I), for assessment of job stress includes job demands (working environment, work overload, time pressures, recipient contact, shift work) and job resources (feedback, rewards, job control, participation, job security, supervisor support) was applied in different studies.

- There is scientific evidence that burned-out physicians have shown depersonalization from their patients, they have withdrawn from patients, demonstrated sub-optimal care, and sometimes burnout has been related to serious mistakes and patient death.

- Different research has shown that some workplace factors contributed to the development of work-related stress and burnout among HPs whereas others contributed protectively.

\section{What does this study add?}

- Similar and overlapping workplace factors in hospital setting produce stress in health professionals and influence quality of care.

- Impact of specific socioeconomic environment in Macedonia as a country in transition and EU candidate country on job stress among health professionals and quality of care.

- Development of criteria and recommendations for the job stress prevention and improvement of the organizational culture and climate in hospital settings.

Health professionals (HPs) are the key actors in the process of implementation of health care measures and activities. They provide all necessary health services to the patients according to their needs and best medical practice in different health care settings. Quality of care is the extent to which health services provided to individuals and patient populations improve desired health outcomes. Health care should be safe, effective, patient-centred, timely, efficient, and equitable (Committee on Quality of Health Care in America \& Institute of Medicine, 2001).

Among numerous hazards at the workplace, HPs are exposed to psychosocial hazards which stem from the workplace conditions and workplace demands. These factors include different aspects of work and work environment, such as organizational climate or culture, interpersonal relationships, design, and content of workplace activities (International Labour Organization [ILO] \& International Occupational Safety and Health Information Centre [CIS], 2000).

Work-related stress can be defined as harmful physical and emotional response that occurs when requirements of the work do not match the capabilities, resources or needs of the worker. Work-related stress can lead to poor health and even injury (National Institute for Occupational Safety and Health [NIOSH], 1999). It can also lead to burnout syndrome, which is often identified among HPs (Leiter \& Maslach, 2000; Maslach \& Leiter, 1997; National Institute for Occupational Safety and Health [NIOSH], 1988).

Workplace stressors affect organizations by increasing absenteeism, decreasing job commitment, increasing staff turnover, impairing performance, and productivity, increasing unsafe working practices, accident rates, complaints from clients, and 
customers including patients (Goldberg et al., 1996; Leiter, Harvie, \& Frizzell, 1998; Leka, Griffiths, \& Cox, 2004; Lemkau, Rafferty, \& Gordon, 1994; NIOSH, 1999; Parker \& Kulik, 1995).

Moreover, stress can have many well-known and detrimental effects on quality of life and work: it might influence overall well-being, social relations, and family life or cause absence from work, early retirement, lower productivity, and lower quality of service or products.

While some studies have analysed associations between burnout and quality of care in hospitals (Bakker, Schaufeli, Sixma, Bosveld, \& Dierendonck, 2000; FirthCozens \& Greenhalgh, 1997; Linn et al., 1986; Montgomery, Panagopoulou, Kehoe, \& Valkanos, 2011; Tait, Shanafelt, Bradley, \& Back, 2002), relations between job stress and quality of care were rarely examined (Klein, Grosse Frie, Blum, \& von dem Knesebeck, 2011).

Our experience concerning work-related stress and burnout syndrome among HPs in the Republic of Macedonia during the last two decades suggested the need for understanding how HPs perceive the link between workplace stressors and quality of care in such socio-economic circumstances.

The specific socio-economic circumstances in the country that resulted from a prolonged process of transition and global crisis, accompanied by the EU accession requests, reflected the national health care system through reforms, new legislation and financing mechanisms, privatization of health care institutions, etc. Therefore, the system faced and still faces multiple challenges in improving access, quality, and efficiency within continuous health care reforms.

To improve the quality of care, social coherence, and democratic sustainability in general, there is still a need for programmatic and social policies with adequate emphasis on stress prevention, improvement in work ability, and prevention of early retirement of HPs.

The current study, conducted by the team from the Institute for Occupational Health of RM-Skopje, WHO Collaborating Centre, was a part of a more comprehensive FP7 Project 'Improving quality and safety in the hospital: The link between organizational culture, burnout, and quality of care (ORCAB)'. The aims of the project were determination of organizational and individual factors that influence quality of care and patients' safety and to design bottom-up interventions that both increase quality of care and HPs' well-being.

\section{Objectives}

The main objective of this article was to identify workplace stressors and factors that influence quality of care from HPs perspective and to recognize their linkages. Additionally, this research was used as a basis for the development of questionnaires for quantitative assessment of work-related stress in HPs and quality of care in hospital settings in the frames of ORCAB Project.

\section{Methods}

Qualitative research using focus group (FG) methodology was conducted in a general teaching hospital in Skopje. The selected hospital provides multidisciplinary work, has a long-term tradition in health care, and has acquired an image of well-organized institution. 
The number of employees was 421, and 162 persons have been working as interns and residents.

The study was conducted between September and November 2010.

The team from the Institute for Occupational Health of RM-Skopje had an official Meeting with the Management Board and Ethical Committee of the Hospital where the project activities were presented.

\section{Focus groups}

After official approval from the hospital management and hospital ethical committee, participants for FGs were recruited. At the beginning of the recruitment, health professionals (HP) from the hospital were informed about the study, research purpose and type. Notification of each FG, with an invitation to participate, was circulated 2 weeks in advance through usual workplace communications (emails, meetings, notice). Those who agreed to participate and were available at arranged times were included in FGs, with technical assistance of the Head nurse.

Focus groups were held in the conference room of hospital, and the participants sat around an oval table to have a clear view of each other throughout the discussion. All of them accepted to participate in the FGs, and each of the participants signed an informed consent form. They were informed about the objectives of the study and about the FG methodology. They were asked to keep anonymity and confidentiality, and permission for audio recording was approved.

The FGs were conducted by two researchers. The aim of facilitator was to lead the group discussion through specific issues, to stimulate the discussion among participants, and to encourage group interaction and exchange of ideas. The assistant facilitator was responsible for taking notes and audio recording the discussions. Besides the discussion, non-verbal communication, tone of voice, and dynamics between group members were observed and noted. Focus groups lasted about $90 \mathrm{~min}$.

Two main topics were the subjects of discussion in FGs: workplace stressors and factors that influence quality of care, from the HPs perspective.

Six FGs were conducted with a total of 56 HPs - three of them focussed the discussion on workplace stressors and the other three focussed on the topic of quality of care. Each FG was comprised of 8-11 participants of the same HP profile (doctors, nurses, or residents) to minimize potential domination of some members and to reduce inhibition of expression in others.

Within the FGs focussed on 'workplace stressors', different issues were discussed, including the amount and quantity of work; relationships with patients, colleagues, and superiors; work environment and equipment; salary and promotions; leadership and management of the hospital; and national health policy.

Within the FGs focussed on 'quality of care', participants had the opportunity to express their opinion about the quality of care discussing the image of the hospital; physical environment and conditions; medical and technical competences (quick and accurate diagnosis, avoidance of medical errors, efficient and effective treatment); HPs' communication with patients; organizational aspects (bureaucracy, waiting times, waiting lines for treatment, surgery or exams, hospital administration, paperwork, standard protocols, and procedures); the role of the health care system and its reform; and future improvement in quality of care.

Focus groups produced a large corpus of data which actually consisted of two separate sets of data. The first set of data was obtained from the first three FGs which focussed on 
the topic 'workplace stressors among HPs'. The second set of data was obtained from the other three FGs which focussed on the topic 'quality of care'. The two data sets were analysed separately.

We used thematic analysis as a method for identifying, analysing and reporting themes within data (Braun \& Clarke, 2006). We started with transcription of audio recordings, and through the process of reading and re-reading, we became familiar with the data. The analysis continued by noting down the initial ideas; coding interesting features of the data in a systematic way across the entire data set, bringing together data relevant to each code; collating codes into potential themes, gathering all data relevant to each potential theme; checking whether the themes work in relation to the coded extracts and the entire data set; creating a thematic map of the analysis; ongoing analysis to refine the specifics of each theme; and generating clear definitions and names for each theme (Braun \& Clarke, 2006).

We have created two maps and compared them. The first map contained themes relating to workplace stressors, and the other was made up of themes regarding the factors that influence the quality of care. We presented the results in Table, then we analysed and interpreted them in relation to the existing literature.

\section{Results}

The demographic and employment characteristics (gender, mean age, tenure, and tenure at the same hospital) of the participants within selected FGs are presented in Table 1.

In the following parts of the article, research findings supported by vivid and compelling examples and identified themes related to workplace stressors and quality of care are presented.

\section{Themes related to workplace stressors}

Health professionals participating in FGs -workplace stressors - reported both negative and positive aspects of their work. They perceived the factors that generated stress as negative or risk factors and those that reduced the causes of stress at work as positive or protective factors.

Table I. General characteristics - demographic and employment data of the participants within selected focus groups

\begin{tabular}{|c|c|c|c|c|c|}
\hline & $N$ & $\begin{array}{l}\text { Mean age } \\
\text { (years) }\end{array}$ & Gender (M/F) & Tenure (years) & $\begin{array}{l}\text { Tenure at the } \\
\text { same hospital } \\
\text { (years) }\end{array}$ \\
\hline \multicolumn{6}{|c|}{ Focus groups (workplace stressors) } \\
\hline Doctors & 9 & $50.1 \pm 7.9$ & $7 / 2$ & $24.1 \pm 8.6$ & $20.2 \pm 9.0$ \\
\hline Nurses & 11 & $46.5 \pm 7.3$ & $1 / 10$ & $25.5 \pm 8.5$ & $24.8 \pm 9.0$ \\
\hline Interns and residents & 8 & $31.4 \pm 8.9$ & $3 / 5$ & $4.1 \pm 2.9$ & $0.5 \pm 0.3$ \\
\hline \multicolumn{6}{|c|}{ Focus groups (quality of care) } \\
\hline Doctors & 8 & $50.6 \pm 8.0$ & $4 / 4$ & $24.1 \pm 7.9$ & $20.8 \pm 9.4$ \\
\hline Nurses & 11 & $43.5 \pm 8.0$ & $0 / 11$ & $22.2 \pm 6.7$ & $19.5 \pm 5.5$ \\
\hline Interns and residents & 9 & $26.9 \pm 3.8$ & $3 / 6$ & $2.2 \pm 2.7$ & $1.3 \pm 2.3$ \\
\hline
\end{tabular}


6 Jovanka Karadzinska-Bislimovska et al.

\section{Negative factors}

Health professionals, participants in FGs, considered work overload, time pressure, long or unpredictable working bours, shift work, and night work as factors responsible for stress at work.

Work overload and working under time pressure were recognized by the following statements:

We have too many patients and too much different work to do, but a low number of personnel.

We have high frequency of patients, decreased number of health professionals and intensive dynamic of work. The work starts in the morning with reporting to the superiors, visiting patients, working in the office, then paperwork for admission or discharge of patients. I feel like I'm torn by duties.

Every day is different; sometimes we do not have time to break for breakfast; the reason is shortage of personnel.

Long working hours, shift work, and night work were also reported as significant workrelated stressors:

We have shift work, on-duty work, and we are working after 16:00. We are also working on weekends.

Work overload and long working hours were noted as sources of stress by the residents as well:

Every morning our work starts at $8 \mathrm{am}$, by visiting patients, writing reports, and then working in the surgical room. We have $4-5$ surgeries a day. We leave the hospital at 5 or $6 \mathrm{pm}$.

All HPs complained mostly about the emotional challenges of working intensively with other people; working with chronic, incurable or dying patients; being responsible for people; and interacting with patients as exhausting job demands.

Some of the doctors said that 'everyday thinking about patients, diagnostics, and treatment', and 'just being responsible for people', are important emotional challenges in HPs.

Doctors explained the stressful aspect of working with dying patients:

It is particularly stressful when the patient is dying and you can't help him, although all necessary was done.

Problems in interacting with patients were described by some of the participants:

Sometimes, after waiting for a long time for a medical examination, patients can provoke conflict situation.

Sometimes patients threaten to inform the media.

It is very stressful to report unpleasant news and pathological findings to the family. 
Arguing with patients when they have inadequate documentation, and they are not able to pay for services.

Most of the participants were concerned about the lack of health care staff; medical equipment; and supplies. According to doctors, 'the shortage of equipment, as well as insufficient and old equipment produces work related stress'.

Where to send the patient for a quick and efficient performance of analysis?

How to explain to the patient that surgery is delayed when there is a lack of supplies.

Some participants were dissatisfied with career stagnation and low opportunity for professional promotion:

We need continuous vocational education and training that will strengthen self confidence; motivation and willingness to realize the activities. But because of deficiency of time and money we are not able to attend international meetings and to visit hospitals in foreign countries.

All the participants were disappointed by the rewards they receive:

The salaries are low, and we have only acknowledgements, compliments, and gratefulness. Low income forces doctors to migrate to private hospitals or to other countries.

Some of the participants explained the work-home interference:

All problems and frustrations we deal with at work we carry back to the home; the whole family experiences these frustrations once again; the family suffers and experiences nervousness.

Most of the HPs considered that bealth care policy and bealth care reforms were not always adequate, and some of the decisions that were adopted could have negative influence on the organization and function of health care system. Frequent changes in legislation and regulations could cause confusion among HP and patients. The problem of adequate financing of public health institutions was emphasized by HPs:

The flat rate that our hospital receives is much lower than the objective needs of the hospital. It is followed by low salaries, deficiency of funding for equipment, supplies and medicines, lack of funds for education and training for employees, as well as for new hiring.

Health professionals also explained that sometimes patients are 'quickly and ineffectively examined' due to administrative, financial or bureaucratic insufficiencies and that situation was perceived as an additional source of work-related stress.

Positive factors

All involved HPs considered that appropriate physical working conditions were important in reducing stress at work. They were satisfied with the suitable space, temperature, light, ventilation, and hygiene in their hospital. 
8 Jovanka Karadzinska-Bislimovska et al.

Visionary well designed, with a great location and functionality, our Hospital is one of the best. All departments are in one building, functioning like one unit. It is necessary to preserve itone of the nurses said with pride.

They believed that the support they had from superiors and co-workers, independence in decision-making, and the excellent interpersonal relationships were important factors that protected them from stress.

Talking about communication and collaboration among colleagues from all departments and team work, they said:

It is something majestic and this is what works best of all in the hospital. There are no conflicts, no separation, and no competitiveness. We are helping each other.

Interns and residents reported:

We are independent in decision making, but if we need help we have support.

All participants agreed that quick and correct diagnosis, therapeutic success, and positive feedback from patients made them happy and fulfilled.

One of the nurses said:

We are health professionals and we are proud of our work. Our profession gives us the opportunity to feel satisfied and fulfilled. When the personnel are careful and polite when the work is quickly and successfully completed, and the patient is pleased and grateful, I feel satisfied.

Happy patient's face and thanks. It means everything to me.

Analysing the first set of data, we have identified a long list of themes and subthemes related to workplace factors that HPs, participants in FGs, perceived as negative factors or stressors: work overload; working under time pressure; long working hours; unpredictable working hours; shift work; night work; emotional challenges of working intensively with other people; problems in interacting with patients; work with chronic, incurable or dying patients; responsibility for people; lack of health care staff; lack of medical equipment; lack of medical supplies; career stagnation; low opportunity for professional promotion and progression; poor pay; disregarding patients in favour of administrative, financial or bureaucratic needs; negative impact of health care policy and socio-economic situation in the country.

The following are workplace factors HPs perceived as positive factors that protected them from stress: physical working conditions (suitable space, temperature, light, ventilation, hygiene); support and feedback from superiors; support and feedback from co-workers; proper participation in decision-making; excellent interpersonal relationships; quick and correct diagnoses; therapeutic success; and positive feedback from patients.

Themes and subthemes related to each other were combined in main themes, and a map was created.

The following Figure 1 represents the map with themes related to the workplace factors that hospital HPs were faced with. 


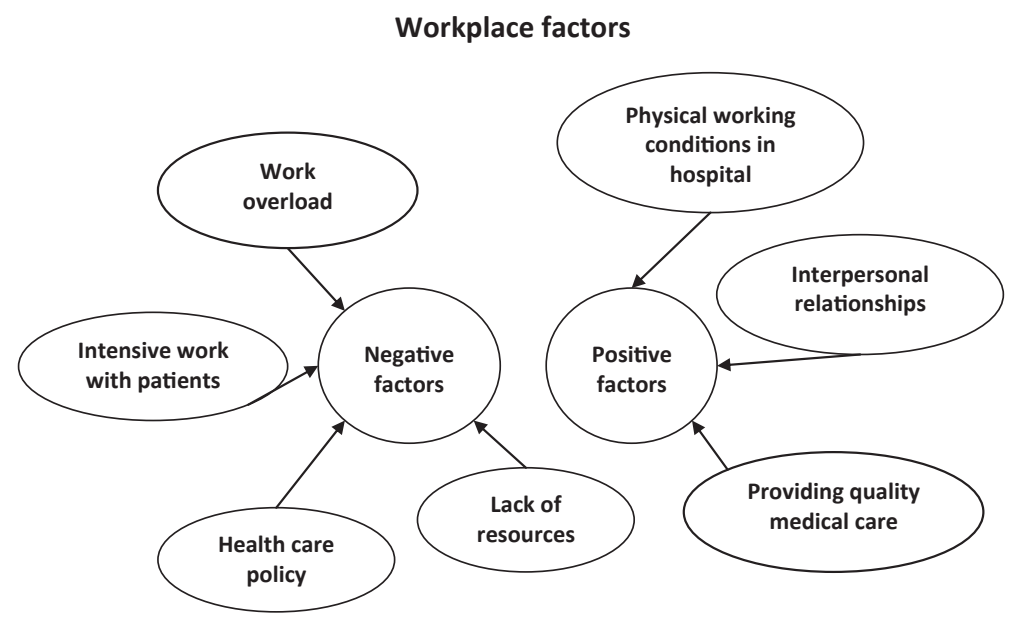

Figure I. Themes related to the workplace factors that hospital health professionals were faced with.

\section{Themes related to the quality of care}

Health professionals participating in three FGs concerning quality of care reported both positive and negative aspects of this issue.

The factors that improved the quality of care were recognized as positive factors and those that reduced the quality of care as negative factors.

\section{Positive factors}

Commenting the quality of care, HPs emphasized the favourable physical conditions for bospitalization (location, bospital facilities, functional connection of all departments, bygiene, and comfort), then the multidisciplinary work, professional and skilled staff, and excellent communication and collaboration among staff from different departments as positive factors that improved quality of care.

In terms of appropriate conditions in the hospital, doctors agreed that 'good capacities, resources, building and construction of the bospital' were necessary in providing health care services. 'Good organization of the hospital and good communication between departments, that is, functionally good building,' were defined as prerequisites for high quality of health care services.

All of the HPs examined agreed that

excellent communication and support between colleagues, correct relationship with patients, discipline and order in work, and high enthusiasm of the personnel' were important factors for good quality of care. 'The patient receives all needed services quickly and efficiently which is enabled by good communication between colleagues and departments.

Support, counselling and training of young colleagues could help to improve the quality of care. One nurse commented: 'It is important to transfer the knowledge and experience to young colleagues, to teach them to be polite, careful and correct with patients, to know to work with patients, not only with equipment'.

The important thing is the reaction of nurses and doctors and nonverbal aspects of their communication. 


\section{Negative factors}

The majority of participants emphasized that old, dysfunctional equipment, lack of resources, insufficient number of HPs, low salaries and some unresolved issues in the national health policy were the factors that had a negative impact on the quality of care.

One of the doctors said: 'How to provide quality care with an insufficient number of employees, with old equipment, with limited amounts of supplies and medicines'.

Health professionals believed that 'low salaries are the reason why experienced staff left the hospital and moved to the private sector'.

In the HPs' opinion, general health care policy had many challenges related to hospital financing, HPs' rights, frequent changes in legislation, and regulations:

Budget set by the health insurance fund is insufficient and does not cover the actual costs.

There is no good care if the Fund approves half of the funds for supplies and if the Ministry has a policy consumes as much as you have.

Where are the doctor's rights? All this smear campaign against doctors encourage some patients to behave inappropriately, anyone can enter the office to attack me verbally or even physically.

Analysing the second set of data, we have identified a list of themes and subthemes related to factors affecting quality of care that HPs, participants in the FGs, perceived as positive or factors that improve the quality of care: respectable conditions in terms of excellent hospital facilities, hygiene and comfort, location of the hospital, functional connection of all departments; multidisciplinary work; professional and skilled staff; excellent communication and collaboration among staff from various departments.

The following themes referred to factors that HPs perceive as negative or factors that reduce the quality of care: old, dysfunctional equipment and limited opportunities for fast and correct diagnosis; lack of resources; insufficient number of HPs (increasing workload, fast pace of work, long working hours, lack of time for professional development); low salaries; negative impact of health care policy and socio-economic situation in the country.

Themes and subthemes from the second set of data, related to each other, were combined in main themes and the second map was created.

Figure 2 represents the map with themes related to the quality of care, most often described by FGs' participants.

In that way, by analysing the data from two different sets of FGs that worked independently of each other, we got two different maps. While preparing the maps, something interesting has attracted our attention. Some of the themes overlapped in both maps. In other words, some of the themes were identical for both topics: 'workplace stressors' and 'factors affecting quality of care'. That was the reason why we decided to compare the maps after the completion of the thematic analysis.

The following themes, related to both negative workplace factors and negative factors reducing quality of care, were present in both maps: lack of staff and large number of patients; lack of equipment; lack of supplies and medications; few opportunities for professional development, lack of training and continual education; low salaries of HPs; and health care policy (Table 2).

The following themes, related to both positive workplace factors and positive factors improving quality of care, were also present in both maps: appropriate environment and 
Factors that influence the quality of care
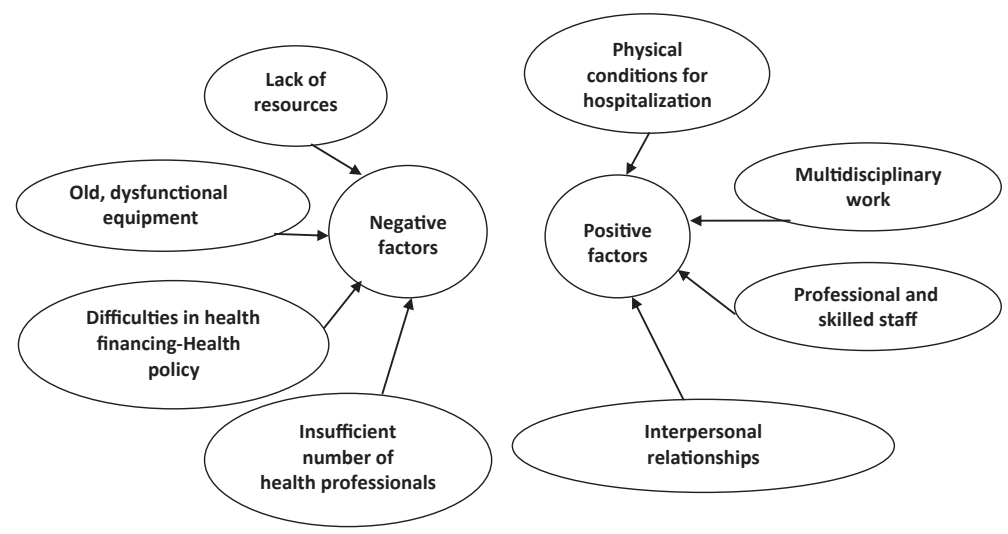

Figure 2. Factors that influence the quality of care.

Table 2. Themes related to both negative workplace factors (stressors) and negative factors reducing quality of care

\begin{tabular}{|c|c|}
\hline Negative workplace factors - stressors & Negative factors reducing quality of care \\
\hline Refers to health workers & Refers to the patients \\
\hline \multicolumn{2}{|l|}{ Lack of staff and a large number of patients } \\
\hline Work overload & $\begin{array}{l}\text { Prolonged waiting time for } \\
\text { examination and admission }\end{array}$ \\
\hline \multicolumn{2}{|l|}{ Increased pace of work } \\
\hline Less time for each patient & Shorter time for conversation \\
\hline Less attention paid to patients & $\begin{array}{l}\text { Less opportunity to explain the } \\
\text { symptoms }\end{array}$ \\
\hline \multicolumn{2}{|l|}{ Lack of equipment } \\
\hline $\begin{array}{l}\text { Limited opportunities for fast and } \\
\text { accurate diagnosis }\end{array}$ & $\begin{array}{l}\text { Harmful delay in examinations and } \\
\text { treatment }\end{array}$ \\
\hline \multicolumn{2}{|l|}{ Increased risk of oversight } \\
\hline \multicolumn{2}{|l|}{ Lack of supplies and medications } \\
\hline $\begin{array}{l}\text { Delay of laboratory and other } \\
\text { diagnostic tests, operations } \\
\text { and treatment }\end{array}$ & $\begin{array}{l}\text { Obligation to provide their own } \\
\text { materials and medicines }\end{array}$ \\
\hline $\begin{array}{l}\text { Inefficiency in the performance } \\
\text { of the work }\end{array}$ & Extra costs and time spent \\
\hline \multicolumn{2}{|c|}{ Few opportunities for professional development, training and continuous vocational education } \\
\hline Uncertainty in the performance of work & Lack of trust in staff \\
\hline Decreased self-confidence & Increased uncertainty about health \\
\hline \multicolumn{2}{|l|}{ Low salaries of health professionals } \\
\hline Increased dissatisfaction & $\begin{array}{l}\text { Few opportunities for patients in } \\
\text { public hospitals to reach top } \\
\text { experts from a certain area }\end{array}$ \\
\hline \multicolumn{2}{|l|}{ Reduced self-esteem } \\
\hline Increased leaving of public health facilities & \\
\hline \multicolumn{2}{|l|}{ Health care policy } \\
\hline $\begin{array}{l}\text { Lack of financial resources for public health } \\
\text { care facilities }\end{array}$ & Lack of funds for health insurers \\
\hline Complex administrative requirements & Complex administrative requirements \\
\hline
\end{tabular}


Jovanka Karadzinska-Bislimovska et al.

Table 3. Themes related to both positive workplace factors and positive factors improving quality of care

\begin{tabular}{|c|c|}
\hline Positive workplace factors & Positive factors improving quality of care \\
\hline Refers to health workers & Refers to the patients \\
\hline \multicolumn{2}{|c|}{$\begin{array}{l}\text { Appropriate environment and suitable physical conditions (space, temperature, lighting, } \\
\text { ventilation, hygiene) }\end{array}$} \\
\hline For work & For hospitalization \\
\hline Motivation for work & Comfortable accommodation \\
\hline Job satisfaction & Satisfaction with services \\
\hline \multicolumn{2}{|c|}{$\begin{array}{l}\text { Good communication and cooperation between health professionals and between } \\
\text { different departments }\end{array}$} \\
\hline $\begin{array}{l}\text { Good opportunities for joint problem } \\
\text { solving, mutual assistance and support }\end{array}$ & $\begin{array}{l}\text { An opportunity for quick resolving } \\
\text { the health problem }\end{array}$ \\
\hline \multicolumn{2}{|c|}{ Correct relationship between health professionals and patients } \\
\hline Satisfied health workers & Satisfied patients \\
\hline & Reduced complaints \\
\hline
\end{tabular}

suitable physical conditions; good communication and cooperation between HPs and between different departments; and correct relationship between HPs and patients (Table 3).

Those findings have suggested that the same factors at the same time were responsible for both stress among HPs and poor quality of care. On the other hand, factors that protected HPs from stress could contribute to better quality of care.

\section{Discussion}

Our study aimed to profile workplace stressors and factors that influence quality of care from HPs' perspective in one Macedonian hospital and to recognize their linkages. The selected hospital has a long-standing tradition in health care and an image of a well-organized institution, which previously was a Military hospital. Nowadays, due to the health care reforms, it is functioning as a general hospital providing care to the general population at the secondary and tertiary level. However, the hospital inherited the previous good organization and management standards, characterized by discipline, hierarchy, teamwork, excellent interpersonal relationships, good communication, and cooperation between HPs and different departments.

This qualitative research was conducted using FGs methods and thematic analysis to identify and interpret issues arising from FGs' discussions in two main topics: workplace stressors and factors that influence quality of care, from the HPs perspective. The factors recognized as identical for both topics were further analysed, and the links between work-related stress and quality of care were made.

Although psychosocial stress at work has been frequently registered among HPs, especially doctors and nurses, the association between job stress and quality of care has not been intensively examined (Klein et al., 2011).

In our research, we utilized the demands/resources model of stress/burnout by Demerouti and Bakker (JD-R model; Demerouti, Bakker, Nachreiner, \& Schaufeli, 2001) where workplace stressors are understood as external factors that have potentials to exert negative influence on most people in most situations. The themes concerning positive and 
negative workplace factors that emerged from the first set of FGs data, referred to job demands and job resources.

The themes emerged from the second set of FGs data concerning factors that influence quality of care were explained by the criteria for quality of care within the theoretical framework given by the Committee on Quality of Health Care in America and Institute of Medicine (Committee on Quality of Health Care in America \& Institute of Medicine, 2001).

In actual research, lack of staff and large number of patients, identified as workplace stressors, leading to work overload, increased pace of work, time pressure, and prolonged working hours were recognized as high job demands. In such conditions, HPs had less time to pay enough attention to patients. Patients had prolonged waiting time for examination and admission to hospital, shorter time for conversation, less opportunity to explain the health problems, which were factors that affect quality of care. The criteria 'timely', 'safe', and 'patient-centred' for quality health care were not met.

Health professionals, also, considered that lack of equipment reduced their opportunities to provide the necessary examinations for diagnosis and increased the risk for medical errors, which was stress for HPs, and decreased efficiency and effectiveness for the quality of care. Patients were referred to other institutions, and examinations and treatment were delayed. These limited job resources have affected the effectiveness, efficiency and timeliness as criteria for quality of care.

Health professionals believed that lack of supplies and medications could delay medical interventions. They felt inefficient in their work. Patients had to provide their own materials and medicines; they had extra costs and time spent. These limited job resources have affected the efficiency and patient centeredness as criteria for quality of care.

Health professionals complained that few opportunities for professional development and lack of training made them uncertain in professional activities and reduced their self-confidence. These limited organizational job resources have contributed for the exposure of patients to ineffective health care.

Health professionals perceived low salaries as stressful factor that was responsible for increased dissatisfaction and reduced self-esteem. Experts left the public health institutions and joined the private sector in search of better earnings. Patients had few opportunities to reach top experts from a certain area in public hospitals. These limited job resources have affected the effectiveness and equity as criteria for quality of care.

Health professionals considered that bealth care policy was not always correct, especially in the case of financing of public health institutions, recognized as limited job resources. It was followed by low salaries, deficiency of funding for equipment, supplies and medicines, lack of funds for education and training for employees, as well as for new employments. Funds for health insurers were limited. Frequent changes in legislation and regulations could cause confusion among HP and patients. Thus, all the criteria 'safe', 'effective', 'patient-centred', 'timely', 'efficient', and 'equitable' for quality health care were not met.

Similarly to our actual research, high job demands (work overload, time pressure, long working hours, shift work) and limited job resources (job insecurity, low potential for qualification, and performance feedback, inadequate rewards) as negative workplace factors were reported as significant workplace stressors in health care settings elsewhere (Aasland, Olff, Falkum, Schweder, \& Ursin, 1997; Eriksen, Tambs, \& Knardahl, 2006; Kinzl, Traweger, Biebl, \& Lederer, 2006; Visser, Smets, Oort, \& De Haes, 2003).

Previous Macedonian studies have also identified work overload and time pressure as well as responsibility for patients, emotional challenges of working with patients, low 
opportunity for promotion, and low support by superiors and colleagues as the most important workplace stressors in HPs (Basarovska, Karadzinska-Bislimovska, Stoleski, \& Mijakoski, 2007; Karadzinska-Bislimovska et al., 2004).

Different qualitative studies reported that lack of human and material resources was significant barriers in providing hospital care. Insufficient rewards and limited opportunities for career development and further education were perceived as significant obstacles for quality of care (Akpalu et al., 2010; Bradley \& McAuliffe, 2009).

Klein et al. (2011) have shown that theoretical models of psychosocial stress at work could enrich the analysis of effects of working conditions on health care quality. Survey data from 1,311 surgeons from 489 hospitals in Germany indicated that high levels of job stress among clinicians could be a risk to physicians' and patients' health. It confirmed the associations between job stress and perceived health care quality.

In our study, despite the negative workplace factors, positive ones were identified and the links with quality of care were elaborated.

Health professionals believed that environment in the bospital was favourable and physical conditions were appropriate. As protective workplace factors, they positively influenced job demands (JD-R model), enhancing motivation and willingness for work, and increasing job satisfaction. Such conditions meant comfortable and safe accommodation for patients. The criteria 'patient-centred', and 'safe' for quality care were met.

Improvements in nurses' work environments in hospitals have the potential to simultaneously reduce nurses' high levels of job stress and increase patients' satisfaction (Vahey, Aiken, Sloane, Clarke, \& Vargas, 2004).

Health professionals perceived good communication and cooperation between bealth professionals and between different departments, as opportunity to help each other and to solve problems together which included absence of conflicts, separation, and competition. These social job resources could protect HPs from stress. For patients, it meant an opportunity for a quick and successful resolving the health problems. The criteria 'effective', 'efficient', and 'timely' for quality care were met.

Health professionals thought that correct relationship between bealth professionals and patients provide a calm and pleasant working atmosphere and could protect them from stress. The JD-R model defines these factors as feedback job resources. When the personnel are careful and polite and when the work is quickly and successfully completed, patients were pleased and grateful. The criterion 'patient-centred' for good quality of health care was met.

Literature showed that job satisfaction, supportive interpersonal relationships, participation in decision-making and teamwork were recognized as protective factors towards work-related stress as in our study (Calnan, Wainwright, Forsythe, Wall, \& Almond, 2001; Le Gall, Azoulay, Embriaco, Poncet, \& Pochard, 2011; Milosevic, 2010; Snelgrove, 1998).

Also, stressed, burned out physicians reported a greater likelihood of making errors and more frequent instance of suboptimal patient care. Therefore, having clinicians who are satisfied and not stressed contributes substantially to the delivery of quality care (Williams, Manwell, Konrad, \& Linzer, 2007).

The results from our study, based on the FG methodology, concerning the link between workplace stressors in HPs and quality of care in selected hospital, are pioneering work on this issue in the country and thus should catalyse further research activities. To have richer and more complex data, it would be useful to include FGs with patients and to compare their comments from the patients' perspective with those from the perspective of HPs. 
Additionally, the more comprehensive results and conclusions on this issue will be provided through quantitative assessment of work-related stress in HPs and quality of care in hospital settings, as the second phase of the ORCAB Project.

\section{Conclusions}

This study has described workplace stressors in hospital setting and factors that have influence on quality of care from HPs' perspective. Results obtained from the current study have given an opportunity to recognize that some of the themes were identical for both topics: workplace stressors and factors affecting quality of care. This means that positive factors that reduce the risk of stress among HPs, at the same time, can improve the quality of care, and negative factors that increase the risk of stress, at the same time, can reduce the quality of care.

Implementation of specific organizational interventions in the hospital setting, and providing adequate job demands-resources interaction, can lead to prevention of workrelated stress and improvement in quality of care.

Therefore, our research suggests that the prevention of work-related stress will impact positively on the quality of care, which may contribute to establish criteria and recommendations for the improvement in organizational culture and climate in hospitals.

\section{Acknowledgement}

The research leading to these results has received funding from the European Union's Seventh Framework Programme [FP7-HEALTH-2009-single-stage] under grant agreement no. [242084].

\section{References}

Aasland, O. G., Olff, M., Falkum, E., Schweder, T., \& Ursin, H. (1997). Health complaints and job stress in Norwegian physicians: The use of an overlapping questionnaire design. Social Science E Medicine, 45, 1615-1629. doi:10.1016/S0277-9536(97)00093-2

Akpalu, B., Lund, C., Doku, V., Ofori-Atta, A., Osei, A., Ae-Ngibise, K., ... MHAPP Research Programme Consortium. (2010). Scaling up community-based services and improving quality of care in the state psychiatric hospitals: The way forward for Ghana. African Journal of Psychiatry, 13, 109-115.

Bakker, A., Schaufeli, W., Sixma, H., Bosveld, W., \& Dierendonck, D. (2000). Patient demands, lack of reciprocity, and burnout: A five-year longitudinal study among general practitioners. Journal of Organizational Behavior, 21, 425-441. doi:10.1002/(SICI)1099-1379(200006)21:4<425::AIDJOB21>3.0.CO;2-\#

Basarovska, V., Karadzinska-Bislimovska, J., Stoleski, S., \& Mijakoski, D. (2007). Surgical nurses, stress at work and burnout syndrome. In E. Kortum (Ed.) (Guest Editor: Carina Ferreira-Borges), GOHNET Special. Addressing psychosocial risks and work-related stress in countries in economic transition, in newly industrialized countries, and in developing countries (pp. 1822). Geneva, Switzerland: World Health Organization (WHO).

Bradley, S., \& McAuliffe, E. (2009). Mid-level providers in emergency obstetric and newborn health care: Factors affecting their performance and retention within the Malawian health system. Human Resources for Health, 7, 14. doi:10.1186/1478-4491-7-14

Braun, V., \& Clarke, V. (2006). Using thematic analysis in psychology. Qualitative Research in Psychology, 3, 77-101. doi:10.1191/1478088706qp063oa 
Calnan, M., Wainwright, D., Forsythe, M., Wall, B., \& Almond, S. (2001). Mental health and stress in the workplace: The case of general practice in the UK. Social Science \& Medicine, 52, 499-507.

Committee on Quality of Health Care in America, \& Institute of Medicine. (2001). Crossing the quality chasm: A new bealth system for the 21st century. Washington, DC: National Academy Press.

Demerouti, E., Bakker, A. B., Nachreiner, F., \& Schaufeli, W. B. (2001). The job demands-resources model of burnout. The Journal of Applied Psychology, 86, 499-512. doi:10.1037/0021-9010.86. 3.499

Eriksen, W., Tambs, K., \& Knardahl, S. (2006). Work factors and psychological distress in nurses' aides: A prospective cohort study. BMC Public Health, 6, 290. doi:10.1186/1471-2458-6-290

Firth-Cozens, J., \& Greenhalgh, J. (1997). Doctors' perceptions of the links between stress and lowered clinical care. Social Science \& Medicine, 44, 1017-1022. doi:10.1016/S0277-9536(96) 00227-4

Goldberg, R., Boss, R. W., Chan, L., Goldberg, J., Mallon, W. K., Moradzadeh, D., ... McConkie, M. L. (1996). Burnout and its correlates in emergency physicians: Four years' experience with a wellness booth. Academic Emergency Medicine, 3, 1156-1164. doi:10.1111/j.15532712.1996. tb03379.x

International Labour Organization, \& International Occupational Safety and Health Information Centre. (2000). Hazard datasbeets on occupations (HDO). Geneva, Switzerland: International Labour Organization (ILO), International Occupational Safety and Health Information Centre (CIS). Retrieved from http://www.ilo.org

Karadzinska-Bislimovska, J., Mijalkov, B., Grunevska, V., Isjanovska, R., Todorov, S., Petrovska, J., ... Mitreski, N. (2004). Specific occupational risks in healthcare workers - Infectious and psychosocial hazards. (Project No 40116101/0). Skopje, Macedonia: Ministry of Education and Science.

Kinzl, J. F., Traweger, C., Biebl, W., \& Lederer, W. (2006). Burnout and stress disorders in intensive care doctors. Deutsche Medizinische Wochenschrift, 131, 2461-2464. doi:10.1055/s-2006955030

Klein, J., Grosse Frie, K., Blum, K., \& von dem Knesebeck, O. (2011). Psychosocial stress at work and perceived quality of care among clinicians in surgery. BMC Health Services Research, 11, 109. doi:10.1186/1472-6963-11-109

Le Gall, J. R., Azoulay, E., Embriaco, N., Poncet, M. C., \& Pochard, F. (2011). Burn out syndrome among critical care workers. Bulletin de l'Académie Nationale de Médecine, 195, 389-397.

Leiter, M. P., Harvie, P., \& Frizzell, C. (1998). The correspondence of patient satisfaction and nurse burnout. Social Science E Medicine, 47, 1611-1617. doi:10.1016/S0277-9536(98)00207-X

Leiter, M. P., \& Maslach, C. (2000). Burnout and health.In A. Baum, T. Revenson \& J. Singer (Eds.), Handbook of health psychology (pp. 415-426). Hillsdale, NJ: Lawrence Earlbaum. Retrieved from http://cord.acadiau.ca/publications.html

Leka, S., Griffiths, A., Cox, T., \& Institute of Work, Health \& Organizations. (2004). Work organization and stress: Systematic problem approaches for employers, managers and trade union representatives. Geneva, Switzerland: World Health Organization (WHO).

Lemkau, J., Rafferty, J., \& Gordon, R., Jr (1994). Burnout and career-choice regret among family practice physicians in early practice. Family Practice Research Journal, 14, 213-222.

Linn, L. S., Brook, R. H., Clark, V. A., Davies, A. R., Fink, A., Kosecoff, J., \& Salisbury, P. (1986). Work satisfaction and career aspirations of internists working in teaching hospital group practices. Journal of General Internal Medicine, 1, 104-108.

Maslach, C., \& Leiter, M. P. (1997). The truth about burnout. San Francisco, CA: Jossey-Bass.

Milosevic, M. (2010). Development and evaluation of the measuring tool for perceived workplace stressors among hospital workers. $\mathrm{PhD}$ thesis. Zagreb, Croatia: University in Zagreb, Medical Faculty.

Montgomery, A., Panagopoulou, E., Kehoe, I., \& Valkanos, E. (2011). Connecting organisational culture and quality of care in the hospital: Is job burnout the missing link? Journal of Health Organization and Management, 25, 108-123. doi:10.1108/14777261111116851 
National Institute for Occupational Safety and Health. (1988). Proposed national strategy for the prevention of psychological disorders (DHHS [NIOSH] Publication No. 89-137). Cincinnati, $\mathrm{OH}$ : National Institute for Occupational Safety and Health (NIOSH). Retrieved from http://www. cdc.gov/niosh/docs/89-137/pdfs/89-137.pdf

National Institute for Occupational Safety and Health. (1999). Stress at work (DHHS [NIOSH] publication no. 99-101). Cincinnati, OH: National Institute for Occupational Safety and Health.

Parker, P. A., \& Kulik, J. A. (1995). Burnout, self- and supervisor-rated job performance, and absenteeism among nurses. Journal of Behavioral Medicine, 18, 581-599. doi:10.1007/ BF01857897

Snelgrove, S. R. (1998). Occupational stress and job satisfaction: A comparative study of health visitors, district nurses and community psychiatric nurses. Journal of Nursing Management, 6 , 97-104. doi:10.1046/j.1365-2834.1998.00055.x

Tait, D., Shanafelt, K. A., Bradley, J. E. W., \& Back, A. L. (2002). Burnout and self-reported patient care in an internal medicine residency program. Annals of Internal Medicine, 136, 358-367.

Vahey, D. C., Aiken, L. H., Sloane, D. M., Clarke, S. P., \& Vargas, D. (2004). Nurse burnout and patient satisfaction. Medical Care, 42 (2 Suppl.), II57-II66. doi:10.1097/01.mlr.0000109126.50398.5a

Visser, M. R., Smets, E. M., Oort, F. J., \& De Haes, H. C. (2003). Stress, satisfaction and burnout among Dutch medical specialists. Canadian Medical Association Journal, 168, 271-275.

Williams, E. S., Manwell, L. B., Konrad, T. R., \& Linzer, M. (2007). The relationship of organizational culture, stress, satisfaction, and burnout with physician-reported error and suboptimal patient care: Results from the MEMO study. Health Care Management Review, 32, 203-212.

Received 30 April 2012; revised version received I February 2013 